\title{
Surgical Treatment of Chronic Parotitis
}

\author{
Rik Johannes Leonardus van der Lans ${ }^{1} \quad$ Peter J.F.M. Lohuis ${ }^{1} \quad$ Joost M.H.H. van Gorp ${ }^{2} \quad$ Jasper J. Quak ${ }^{1}$ \\ ${ }^{1}$ Department of ENT \& Head and Neck Surgery, Diakonessenhuis, \\ Utrecht, Netherlands \\ 2 Department of Pathology, Diakonessenhuis, Utrecht, Netherlands \\ Address for correspondence Rik Johannes Leonardus van der Lans, \\ MD, Department of ENT- \& Head and Neck Surgery, Diakonessenhuis, \\ location Utrecht, Bosboomstraat 1, 3582 KE, Utrecht, Netherlands \\ (e-mail: rjlvanderlans@gmail.com).
} Int Arch Otorhinolaryngol 2019;23:83-87.

\begin{abstract}
Keywords

- parotid gland

- sialadenitis

- treatment outcome

Introduction chronic parotitis (CP) is a hindering, recurring inflammatory ailment that eventually leads to the destruction of the parotid gland. When conservative measures and sialendoscopy fail, parotidectomy can be indicated.

Objective to evaluate the efficacy and safety of parotidectomy as a treatment for CP unresponsive to conservative therapy, and to compare superficial and near-total parotidectomy (SP and NTP).

Methods retrospective consecutive case series of patients who underwent parotidectomy for CP between January 1999 and May 2012. The primary outcome variables were recurrence, patient contentment, transient and permanent facial nerve palsy and Frey syndrome. The categorical variables were analyzed using the two-sided Fisher exact test. Alongside, an elaborate review of the current literature was conducted.

Results a total of 46 parotidectomies were performed on 37 patients with CP. Neartotal parotidectomy was performed in 41 and SP in 5 cases. Eighty-four percent of patients was available for the telephone questionnaire (31 patients, 40 parotidectomies) with a mean follow-up period of 6,2 years. Treatment was successful in 40/46 parotidectomies $(87 \%)$ and $95 \%$ of the patients were content with the result. The incidence of permanent and transient facial nerve palsy was $0(0 \%)$ and $12(26.1 \%)$, respectively. Frey syndrome manifested in $20(43.5 \%)$ patients. Neither this study nor careful review of the current literature resulted in evident difference between SP and NTP regarding the primary outcome variables.

Conclusion parotidectomy is a safe and effective treatment for CP in case conservative therapy fails. There is no evidence of a distinct difference between SP and NTP regarding efficiency, facial nerve palsy or Frey syndrome.
\end{abstract}

\section{Introduction}

The pathophysiological mechanism of chronic parotitis (CP) is considered to be multifactorial, in which glandular duct obstruction and inflammation play a central role. Local causes of reduced salivary flow include sialoliths, anomalies and strictures of the duct or ductal orifice, foreign bodies and external pressure on the Stensen duct. ${ }^{1}$ Dehydration, radiation damage (including radioactive iodine [I-131]) and immune mediated disease (for example, Sjögren disease) are other

received

January 16, 2018

accepted

May 20, 2018

published online

October 24, 2018 10.1055/s-0038-1667006. ISSN $1809-9777$. known causes. ${ }^{2}$ Histologically, $\mathrm{CP}$ is characterized by periductal inflammation, with epithelial proliferation and metaplasia of the duct epithelium and an interepithelial infiltration with lymphocytes. More advanced stages show marked infiltration of chronic inflammatory cells with variable dilatation of the intraglandular ducts. Eventually, acinar atrophy, fibrosis and fatty tissue depositions develop as the lobular structure is progressively destroyed. ${ }^{2-5}$ Treatment of $\mathrm{CP}$ is initially performed with conservative measures to abate and prevent symptoms, including sialogogues, gland massage, analgesics
Copyright @ 2019 by Thieme Revinter Publicações Ltda, Rio de Janeiro, Brazil
License terms

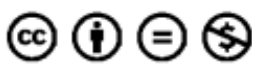


and antibiotics. Unfortunately, conservative management fails in up to half the patients, in which case more resolute therapy is required. ${ }^{1}$ Parotidectomy has been proven to be an effective and save procedure for patients with intractable symptoms. $^{2,6-8}$ In literature on parotidectomy, CP is being reported to be the reason in a considerable percentage of the study population, which is not reflected in the small amount of literature on this topic. Concern of facial nerve paralysis withholds more widespread usage when necessitated. In addition, there is an ongoing debate about the extent of the parotidectomy-either near-total or superficial parotidectomy (NTP or SP)-with regard to safety and efficacy. This study's objective is to both report on and discuss the safety and efficacy of parotidectomy for chronic parotitis with special detail to the surgical extent, by analysis of the presented author's consecutive case series as well as an elaborate review of the current literature on this topic.

\section{Method}

\section{Patients}

All data of patients undergoing parotidectomy at a secondary referral center between January 1999 and May 2012 were collected using patient files and the Dutch national database for pathology. Patients with a clinical diagnosis of CP were included (see - Table 1). Patient characteristics, preoperative diagnostic investigations, type of surgery and the following primary treatment outcomes were extracted: facial nerve dysfunction, Frey syndrome (FS) and recurrence. Recurrence was defined as postoperative symptoms of sialadenitis, confirmed by MRI or ultrasound. To prolong the follow-up (FU) and thus more accurately report the incidence regarding FS

Table 1 Clinical and histopathological definition of chronic parotitis

\begin{tabular}{|c|c|}
\hline \multicolumn{2}{|l|}{ Clinical diagnosis: } \\
\hline \multicolumn{2}{|c|}{$\begin{array}{l}\text { Intermittent bouts of inflammation of the parotid gland, uni- } \\
\text { or bilateral, minimum of } 1 \text { year of conservative treatment or } \\
\text { at least } 4 \text { courses of oral antibiotics without lasting benefit } \\
\text { and exclusion of other (causing) disorders. }\end{array}$} \\
\hline \multirow[t]{2}{*}{ Main symptoms: } & - swelling \\
\hline & - pain/discomfort \\
\hline \multirow[t]{2}{*}{ Accompanying symptoms: } & - erythema of skin \\
\hline & - mucopurulent discharge \\
\hline \multicolumn{2}{|l|}{ Histopathological diagnosis: } \\
\hline \multicolumn{2}{|l|}{ Active stage: } \\
\hline \multicolumn{2}{|c|}{$\begin{array}{l}\text { - Periductal inflammation with infiltration of (chronic) } \\
\text { inflammatory cells }\end{array}$} \\
\hline \multicolumn{2}{|c|}{ - Epithelial proliferation and metaplasia } \\
\hline \multicolumn{2}{|c|}{$\begin{array}{l}\text { Advanced stage (can be observed } w / \text { and } w / o \text { active stage } \\
\text { findings): }\end{array}$} \\
\hline \multicolumn{2}{|c|}{ - Variable dilatation of intraglandular ducts } \\
\hline \multicolumn{2}{|c|}{$\begin{array}{l}\text { - Acinar atrophy with profound fibrosis and/or deposition } \\
\text { of fatty tissue }\end{array}$} \\
\hline
\end{tabular}

and recurrence-both treatment outcomes that can manifest after the clinical FU-a standardized telephone questionnaire was executed, in which we also informed about overall patient satisfaction.

A review of the current literature regarding surgical treatment of chronic parotitis was performed, using PubMed. Extraction of fore mentioned primary treatment outcomes was executed and analyzed, with sub-analysis of surgical extent. This study was approved by the institutional review board (IRB) of the Medical research Ethics Committees United, in The Netherlands, (MEC-U).

\section{Surgical Technique}

The surgical procedure in all cases was initiated with a preauricular lazy-S-shaped incision. The trunk of the facial nerve and all its terminal branches were identified and preserved using atraumatic dissection techniques. Electromyographic facial nerve monitoring has been part of standard care since 2005. After securing the facial nerve, the inflamed parotid tissue was removed. In most cases, to prevent recurrence, a near-total parotidectomy (NTP) was performed after excision of the entire superficial lobe, and as much glandular tissue as possible was carefully removed from the deep lobe between the facial nerve branches. In some cases, superficial parotidectomy (SP) was used. This was either because the inflammation appeared to be defined to the superficial lobe during intraoperative inspection, or because more extensive surgery would bring about too great a risk of facial nerve damage as a consequence of the inflammation and fibrosis.

\section{Data Analysis}

All data analyses were performed using the SPSS Statistics for Windows, Version 17.0 (SPSS Inc., Chicago, IL, USA). Categorical variables were analyzed using the two-sided Fisher exact test. A $p$-value $\leq 0.05$ was considered to be of statistical significance.

\section{Results}

Between January 1999 and May 2012, 46 out of a 382 parotidectomies (12\%) were performed in 37 patients because of intractable CP (-Table 2). The mean age at surgery was 49 years (range 16-80 years), and 24 (65\%) patients were female. Nine patients $(39.1 \%)$ were successively operated bilaterally, of whom only one was male. Neartotal parotidectomy was performed 41 times (89.1\%). In 5 cases $(10.9 \%)$ the deep lobe was left in place. Preoperative diagnostic investigations were performed in all but 2 patients, namely ultrasound (US: 36), computed tomography (CT) scan (8), magnetic resonance imaging (MRI: 11), fine needle aspiration cytology (10), scintigraphy (3) and plain projectional radiography for sialoliths (1). None of the patients in this retrospective study was subject to preceding sialendoscopy.

There was no occurrence of paralysis of the facial nerve (-Table 3). Following 12 parotidectomies, paresis was noted (26.1\%), with a mean duration of 7.7 weeks (range $1-12 w)$. 
Table 2 Patient characteristics

\begin{tabular}{|c|l|}
\hline Patients: & 37 \\
\hline Gender & \\
\hline Male & 13 \\
\hline Female & 24 \\
\hline Age at surgery & \\
\hline Mean & 49 \\
\hline Range & $16-80$ \\
\hline Parotidectomies & 46 \\
\hline Surgeon & \\
\hline PL & 6 \\
\hline JQ & 40 \\
\hline Side & \\
\hline Unilateral & 28 \\
\hline Bilateral & 9 \\
\hline Type of parotidectomy & \\
\hline Superficial & 5 \\
\hline Near total & 41 \\
\hline
\end{tabular}

Postoperative wound infection was found in one patient (2.2\%). Postoperative bleeding unfolded in another patient (2.2\%), which resolved without surgical intervention. Six patients were not available for the telephone questionnaire, two of whom were known to be deceased. In the clinical FU of all 37 patients (median $19 \mathrm{~m}$; range $4-57 \mathrm{~m}$ ), no recurrence or FS was reported. A total of 31 patients $(80 \%)$ was available for the telephone questionnaire, providing up to date information about the postoperative course of 40 parotidectomies (86.9\%). The consequent mean period of FU for all 37 patients was 5.6 years. After parotidectomy, 25 out of 31 patients were symptom-free. Recurrence was reported by 6 cases (15\%; NTP: SP = 3:3), which could be sufficiently treated with conservative treatment in 4 patients. No recurrence had been reported by the patients lost to FU (median 19 months; 4-57). Frey syndrome was reported after 20 parotidectomies, of which only 3 instances were reported during the initial clinical FU period $(6.5 \%$ in mean of 25.8 months). No significant association with surgical extent and either facial nerve dysfunction or recurrence or FS was found ( $p$ respectively $1.0,0.58$ and 0.64 ). Almost $95 \%$ of the patients (29/31) were satisfied with the treatment results. Both patients who were not content had recurring sialadenitis after parotidectomy; one required additional surgery of the deep lobe, and the other opted not to undergo secondary surgery. In all patients, histopathologic examination of the excised glandular tissue confirmed chronic sialadenitis.

\section{Discussion}

This study shows parotidectomy to be a safe and effective treatment for chronic recurring parotitis. After failure of conservative therapy, $80 \%$ of the patients was completely symptom-free after surgery. This is reflected in a patient satisfaction rate of $95 \%$. However, some issues remain regarding parotidectomy for $\mathrm{CP}$ in general and specifically on the subject of SP vs NTP techniques, which are discussed in the next paragraphs.

Table 3 Results and literature overview

\begin{tabular}{|c|c|c|c|c|c|c|c|c|c|c|c|c|c|c|c|c|}
\hline \multirow[t]{2}{*}{ 1st Author } & \multirow[t]{2}{*}{ Year } & \multicolumn{2}{|c|}{ Inclusion } & \multirow[t]{2}{*}{ FU } & \multicolumn{4}{|c|}{ Surgical technique } & \multicolumn{4}{|c|}{ Facial nerve } & \multicolumn{2}{|l|}{ FS } & \multicolumn{2}{|c|}{ Recurrence } \\
\hline & & Pt's & Par. & & $S P$ & $\%$ & NTP & $\%$ & N7P & $\%$ & N7T & $\%$ & $n$ & $\%$ & $n$ & $\%$ \\
\hline Beahrs $^{10}$ & 1961 & 29 & 30 & 6 & 23 & 76.7 & 7 & 23.3 & 0 & 0 & 5 & 17.2 & - & - & 1 & 3.3 \\
\hline Perzik $^{11}$ & 1961 & 38 & 33 & - & 19 & 57.6 & 14 & 42.4 & 0 & 0 & 33 & 100 & 9 & 27.3 & 0 & 0 \\
\hline Nichols $^{12}$ & 1977 & 14 & 14 & 2 & 12 & 85.7 & 2 & 14.3 & 0 & 0 & 6 & 42.9 & - & - & 1 & 7.1 \\
\hline Casterline $^{13}$ & 1978 & 26 & 28 & - & 11 & 39.3 & 17 & 60.7 & 0 & 0 & 18 & 64.3 & - & - & 1 & 3.6 \\
\hline Cancura $^{14}$ & 1982 & 14 & 14 & 5.1 & 7 & 50.0 & 7 & 50.0 & 0 & 0 & 6 & 42.9 & 1 & 7.1 & 0 & 0 \\
\hline Chilla $^{15}$ & 1982 & 58 & 58 & - & 17 & 29.3 & 41 & 70.7 & 7 & 12.1 & 27 & 46.6 & - & - & 6 & 10.3 \\
\hline Schultz $^{16}$ & 1983 & 21 & 24 & 6 & 21 & 87.5 & 3 & 12.5 & 0 & 0 & - & - & 3 & 12.5 & 3 & 12.5 \\
\hline Arriaga $^{17}$ & 1990 & 14 & 16 & 4.8 & 0 & 0 & 16 & 100 & 0 & 0 & 5 & 31.3 & 3 & 18.8 & 2 & 12.5 \\
\hline Bates $^{18 *}$ & 1998 & 47 & 49 & - & 14 & 28.6 & 35 & 71.4 & 1 & 2.0 & 14 & 28.6 & - & - & 1 & 2.0 \\
\hline Bhatty $^{3}$ & 1998 & 19 & 17 & 1.5 & 17 & 100 & 0 & 0 & 0 & 0 & 13 & 76.5 & 3 & 17.6 & 2 & 11.8 \\
\hline Moody ${ }^{1}$ & 2000 & 51 & 47 & 1 & 46 & 97.9 & 1 & 2.1 & 0 & 0 & 28 & 59.6 & 5 & 10.6 & 5 & 10.6 \\
\hline $\mathrm{Amin}^{5}$ & 2001 & 22 & 23 & 4.2 & 23 & 100 & 0 & 0 & 0 & 0 & 15 & 65.2 & 7 & 30.4 & 3 & 13.0 \\
\hline Nouraei $^{7}$ & 2006 & 34 & 36 & 2.9 & 32 & 88.9 & 4 & 11.1 & 1 & 2.8 & 20 & 55.6 & 8 & 22.2 & 6 & 16.7 \\
\hline Patel $^{* 8}$ & 2007 & 75 & 78 & - & 17 & 21.8 & 61 & 78.2 & 1 & 1.3 & 26 & 33.3 & - & - & 2 & 2.6 \\
\hline Sharma $^{2}$ & 2012 & 21 & 21 & 1.7 & 21 & 100 & 0 & 0 & 0 & 0 & 6 & 28.6 & 3 & 14.3 & 0 & 0 \\
\hline This study & a & 37 & 46 & 5.6 & 5 & 10.9 & 41 & 89.1 & 0 & 0 & 12 & 26.1 & 20 & 43.5 & 6 & 13.0 \\
\hline & b & 31 & 40 & 6.2 & 4 & 10.0 & 36 & 90.0 & $\mathrm{x}$ & $\mathrm{x}$ & $\mathrm{x}$ & $x$ & 20 & 50.0 & 6 & 15.0 \\
\hline
\end{tabular}

Abbreviations: (-), not mentioned; (a), results calculated for all patients; (b), results calculated for respondents of telephone questionnaire; FS, Frey syndrome; FU, mean follow-up in years; N7P/N7T, permanent/transient facial nerve palsy; NTP, near-total parotidectomy; Par, number of parotidectomies; Pt's, patients included; SP, superficial parotidectomy.

*: Overlap of study population; 
No clear definition of $\mathrm{CP}$ is stated in the literature regarding duration and severity of symptoms. This impedes a clear comparison of study results. Our requisite to consider surgical intervention is outlined in - Table 1, besides, of course, the willingness of the patient to undergo surgery.

Due to the chronic inflammation and subsequent fibrosis, parotidectomy in $\mathrm{CP}$ is a challenging surgical procedure in which meticulous preparation of the facial nerve is required. A high level of experience in parotid surgery is essential. No permanent facial nerve dysfunction occurred in our series. Within 15 studies, including this one, permanent facial nerve paralysis occurred in $1.9 \%(9 / 485)$ of the cases. ${ }^{1-3,5-7,9-17}$ The majority being reported in 1 single series, by Chilla et al in 1986 $(n=7)$, and 2 other series both noted 1 case. $^{6,7,14,17}$ This considered, the risk of permanent facial nerve paralysis conducting parotidectomy for $\mathrm{CP}$ is comparable to parotidectomy for other benign parotid pathology (up to $4.0 \%$ ). ${ }^{18,19}$ Transient paresis of the facial nerve occurred in $26.1 \%$ of our patients, most often of the oral branch. This corresponds well with other studies. Mixed results are found in the literature as to whether $\mathrm{CP}$ is associated with a higher incidence of transient facial nerve paresis compared with other benign diseases. ${ }^{2,7,17}$ Frey syndrome was reported after $50 \%$ of parotidectomies in our series, comparable to the incidence after parotidectomy for benign parotid disease in general. ${ }^{20}$ The literature on parotidectomy for $\mathrm{CP}$ is fairly consistent regarding recurrence rate, $\sim 15 \%$, which is in line with our findings. ${ }^{1-3,6,14,15}$ Preoperative diagnostic investigation is of importance to gain supportive information about possible underlying causes and to rule out other pathologies, and mostly comprises (a combination of) US, CT and MRI. The previously mentioned diagnostic investigations remain supportive. However, the frequency and severity of episodes, effect of conservative measures as well as disease burden should all be accounted for when indicating surgery. ${ }^{21}$ Other diagnostic techniques include salivary scintigraphy and sialendoscopy. ${ }^{22}$ Salivary endoscopy is also performed as treatment for $\mathrm{CP}$, by removing scar tissue, debris and stones, sometimes combined with saline or steroid irrigations. Promising mid-term success rates are achieved, bearing in mind that this applies to a heterogeneous group of patients with obstructive sialadenitis without distinction in disease severity or duration..$^{23}$ Parotidectomy is sometimes needed when sialendoscopy proves to be insufficient. ${ }^{24}$ Overall, sialendoscopy appears to be of use as a first means of invasive therapy, especially in mild, short-termed disease, whereas parotidectomy can be the designated therapy in case of failure or in more severe cases. As to our knowledge, there are no published studies specifically discussing parotidectomy after failure of sialendoscopy.

With regard to surgical extent, recurrence is most likely caused by residual parotid tissue after parotidectomy. There is an ongoing debate about the surgical extent, with a distinction made between superficial (SP) and near-total parotidectomy (NTP). Proponents of SP state that resection of the lateral lobe already removes the greater part of the affected gland, with less risk for facial nerve damage, better cosmesis and satisfactory therapeutic results. ${ }^{3,5,6}$ Proponents of NTP regard removal of as much glandular tissue as possible of utmost importance for a definitive cure and refute the higher risk of facial nerve damage. ${ }^{1,7,9,14,15,17}$ From an anatomic-surgical standpoint, it is questionable if NTP and SP can truly be distinguished. A study with adequate pre and postoperative imaging-preferably MRI-would be needed to objectify if significant difference between both techniques exists in extent and excised volume.

Regarding permanent facial nerve paralysis, Bates et al and Patel et al-overlapping in study population-report on one case, without stating the surgical technique., ${ }^{77}$ Nouraei et al report one case in which SP was used. ${ }^{6}$ Chilla et al make note of 7 cases: in 3/17 NTP was used, and in $4 / 41 \mathrm{SP}$ was used. ${ }^{14}$ When analyzed, neither of these two studies show significant association between surgical extent and facial nerve paralysis $(p>0.3)$. With regard to transient facial nerve palsy, no significant association was found with surgical extent in the current series. Neither was it found by Patel et al $(p>0.05){ }^{7}$ Two other studies provided sufficient information for statistical analysis: the study by Casterline et al showed inferior results for NTP (relative risk (RR): $2.27 ; p=0.02$ ), while no association resulted from the study by Cancurra et al $(p>0.7) .^{12,13}$ Overall, there is no conclusive evidence for significant differences between NTP and SP in facial nerve damage, be it permanent or temporary.

With regard to treatment outcome, we did not find a significant difference between SP and NTP. Nouraei et al also reported no association between the extent of surgery and recurrence $(p>0.3){ }^{6}$ Nor did 3 other studies providing sufficient information for statistical analyses $(p>0.1){ }^{7,9,12}$

Regarding FS, a significant positive correlation with the extent of surgery is observed in the literature on benign parotid disease. ${ }^{20}$ We did not find such a correlation in our series. Studies included in the literature review did not assess this correlation.

It is questionable whether NTP and SP can truly be discerned and reviewing the literature, no evident differences in outcomes are found. From an etiological perspective, we consider that the key to successful treatment is removal of as much affected glandular tissue as possible, while maintaining integrity of the facial nerve. This view is shared by the majority of authors on this subject. ${ }^{1,7,9,14,15,17}$

Regarding patient satisfaction, we consider this to be of importance as a patient-centered subjective reflection of overall result of the surgery. Treatment outcome after a relative short FU period as documented by the surgeon alone does not fully reflect the effect of the surgery, as a technically satisfying procedure can result in unsatisfactory results for the patient, and vice versa. Despite symptoms not being completely resolved in all patients, overall patient satisfaction appeared to be quite good: $95 \%$ of patients were satisfied with the results. This information is of importance in future surgical indication and counseling of patients.

\section{Conclusion}

Both the presented consecutive case series and an elaborate review of the current literature proves parotidectomy to be a safe and effective treatment for hindering chronic parotitis. There is a low risk of permanent and transient facial nerve 
palsy; the incidence of FS is comparable to that of parotidectomy for other benign pathologies. Recurrence occurs in $\sim 15 \%$ of patients, and even though complete resolution of symptoms could not be achieved in all of them, a high patient satisfaction rate was achieved. No evident difference between SP and NTP resulted from analysis of our case series, or from careful review of the literature. On an etiological basis, it is the authors' view that the key to successful treatment is removal of as much affected glandular tissue as possible, while maintaining integrity of the facial nerve.

\section{References}

1 Moody AB, Avery CM, Walsh S, Sneddon K, Langdon JD. Surgical management of chronic parotid disease. Br J Oral Maxillofac Surg 2000;38(06):620-622. Doi: 10.1054/bjom.2000.0478

2 Sharma R. Superficial parotidectomy for chronic parotid sialadenitis. Int J Oral Maxillofac Surg 2013;42(01):129-132. Doi: 10.1016/j. ijom.2012.04.003

3 Bhatty MA, Piggot TA, Soames JV, McLean NR. Chronic nonspecific parotid sialadenitis. Br J Plast Surg 1998;51(07):517-521

4 Seifert G. Aetiological and histological classification of sialadenitis. Pathologica 1997;89(01):7-17

5 Amin MA, Bailey BM, Patel SR. Clinical and radiological evidence to support superficial parotidectomy as the treatment of choice for chronic parotid sialadenitis: a retrospective study. Br J Oral Maxillofac Surg 2001;39(05):348-352. Doi: 10.1054/bjom.2001.0671

6 Nouraei SA, Ismail Y, McLean NR, Thomson PJ, Milner RH, Welch AR. Surgical treatment of chronic parotid sialadenitis. J Laryngol Otol 2007;121(09):880-884. Doi: 10.1017/S0022215106005445

7 Patel RS, Low T-HH, Gao K, O'Brien CJ. Clinical outcome after surgery for 75 patients with parotid sialadenitis. Laryngoscope 2007;117(04):644-647. Doi: 10.1097/mlg.0b013e318030acc0

8 Harbison JM, Liess BD, Templer JW, Zitsch RP III, Wieberg JA. Chronic parotitis: a challenging disease entity. Ear Nose Throat J 2011;90(03):E13-E16

9 Beahrs OH, Devine KD, Woolner LB. Parotidectomy in the treatment of chronic sialadenitis. Am J Surg 1961;102:760-764

10 Perzik SL. Parotidectomy for inflammatory lesions. Am J Surg 1961 Dec;102:769-776
11 Nichols RD. Surgical treatment of chronic suppurative parotitis. A critical review. Laryngoscope 1977;87(12):2066-2081. Doi: 10.1288/00005537-197712000-00013

12 Casterline PF, Jaques DA. The surgical management of recurrent parotitis. Surg Gynecol Obstet 1978;146(03):419-422

13 Cancura W. [Surgical therapy of chronic parotitis]. Laryngol Rhinol Otol (Stuttg) 1982;61(12):683-685

14 Chilla R, Meyfarth HO, Arglebe C. [Surgical treatment of chronic parotitis (author's transl)]. Arch Otorhinolaryngol 1982;234(01): 53-63

15 Schultz PW, Woods JE. Subtotal parotidectomy in the treatment of chronic sialadenitis. Ann Plast Surg 1983;11(06):459-461

16 Arriaga MA, Myers EN. The surgical management of chronic parotitis. Laryngoscope 1990;100(12):1270-1275

17 Bates D, O'Brien CJ, Tikaram K, Painter DM. Parotid and submandibular sialadenitis treated by salivary gland excision. Aust N Z J Surg 1998;68(02):120-124

18 Yuan X, Gao Z, Jiang H, et al. Predictors of facial palsy after surgery for benign parotid disease: multivariate analysis of 626 operations. Head Neck 2009;31(12):1588-1592. Doi: 10.1002/ hed. 21134

19 Guntinas-Lichius O, Klussmann JP, Wittekindt C, Stennert E. Parotidectomy for benign parotid disease at a university teaching hospital: outcome of 963 operations. Laryngoscope 2006;116 (04):534-540. Doi: 10.1097/01.mlg.0000200741.37460.ea

20 Koch M, Zenk J, Iro H. Long-term results of morbidity after parotid gland surgery in benign disease. Laryngoscope 2010;120(04): 724-730. Doi: 10.1002/lary.20822

21 Zenk J, Iro H, Klintworth N, Lell M. Diagnostic imaging in sialadenitis. Oral Maxillofac Surg Clin North Am 2009;21(03): 275-292. Doi: 10.1016/j.coms.2009.04.005

22 Heineman TE, Kacker A, Kutler DI. Idiopathic Chronic Parotitis: Imaging Findings and Sialendoscopic Response. ORL J Otorhinolaryngol Relat Spec 2015;77(05):302-309. Doi: 10.1159/000438760

23 Strychowsky JE, Sommer DD, Gupta MK, Cohen N, Nahlieli O. Sialendoscopy for the management of obstructive salivary gland disease: a systematic review and meta-analysis. Arch Otolaryngol Head Neck Surg 2012;138(06):541-547. Doi: 10.1001/archoto.2012.856

24 Gillespie MB, O'Connell BP, Rawl JW, McLaughlin CW, Carroll WW, Nguyen SA. Clinical and quality-of-life outcomes following glandpreserving surgery for chronic sialadenitis. Laryngoscope 2015; 125(06):1340-1344. Doi: 10.1002/lary.25062 